\title{
Revelación de lineamientos sobre responsabilidad social en los códigos de buen gobierno de las empresas cotizadas en Colombia*
}

doi: 10.11144/Javeriana.cc15-38.rlsr

\section{Laura Tatiana Romero-González}

Contadora pública, Universidad de La Salle. Miembro del semillero de investigación Responsabilidad, rendición de cuentas y transparencia.

Correo electrónico: laura8romero25@gmail.com

\section{Luisa Fernanda Wanumen-Marín}

Contadora pública, Universidad de La Salle. Miembro del semillero de investigación Responsabilidad, rendición de cuentas y transparencia.

Correo electrónico: luisafer.wanumenmarin@gmail.com

\begin{abstract}
Diego Fernando Católico-Segura
Contador público, Universidad Nacional de Colombia. Especialista en finanzas, Universidad Santo Tomás. Magíster en contabilidad y finanzas, Universidad de Zaragoza, España. Docente-investigador, Universidad de La Salle. Líder del grupo de investigación Responsabilidad, rendición de cuentas y transparencia.
\end{abstract}

Correo electrónico:dfcatolico@unisalle.edu.co

\footnotetext{
* Artículo derivado de un proceso de investigación que se adelantó bajo la modalidad de grado producción intelectual relevante, avalada por el programa de contaduría pública de la Universidad de La Salle. La propuesta de la modalidad de grado fue Revelación de los lineamientos sobre responsabilidad social en los códigos de buen gobierno en las empresas cotizadas en Colombia, comenzó en agosto de 2012 y terminó en noviembre de 2013.
} 
Resumen En el presente artículo se estudia la revelación de lineamientos sobre responsabilidad social en los códigos de buen gobierno de las empresas que cotizan en el mercado público de valores de Colombia. Para ello, se identificaron los códigos de buen gobierno publicados en los sitios web de las empresas que conforman la canasta del Índice General de la Bolsa de Valores de Colombia, IGBC, vigente para el segundo trimestre de 2013; se indagaron los aspectos que son propios de las mejores prácticas corporativas y aquellos asociados con los lineamientos de la responsabilidad social empresarial, RSE, en particular, los principios, la relación con los grupos de interés, las estrategias y actividades, y la revelación y divulgación de información, entre otros. A partir de lo anterior, se evidencia que las empresas analizadas no dan cuenta de la adopción de lineamientos de responsabilidad social por medio de los códigos de buen gobierno, lo que privilegia en mayor medida la revelación de información en temas de estructura organizacional y financieros, que son obligatorios y necesarios para los inversores bursátiles o acreedores financieros.

Palabras clave Gobierno corporativo; empresas cotizadas; responsabilidad social; revelación y divulgación de la información

\section{Código JEL M 14, M 41}

\section{Revealing the Guidelines on Social Responsibility in the Good Governance Codes of the Listed Companies in Colombia}

\footnotetext{
Abstract This article studies the revealing of the guidelines on social responsibility in the good governance codes of the publicly listed companies in the stock market in Colombia. For this purpose we identified the good governance codes published in the websites of the companies that make up the group of the General Stock Market Index of Colombia - IGBC_ - valid for the second quarter of 2013. We researched on several aspects typical to the best corpo-
}

rate practices and those related to the guidelines of corporate social responsibility, RSE, particularly the principles, the relationship with the stakeholders, the strategies and activities, and the disclosure and spreading of information, among others. From this, it is evident that the analyzed companies do not account for the adoption of guidelines of social responsibility through the codes of good governance. This favors the most the disclosure of information on topics of organizational structure and finance, which are necessary and mandatory for market investors or financial creditors.

Keywords company governance; publicly-listed companies; social responsibility; disclosure and spread of information

\section{Revelação dos diretrizes sobre responsabilidade social nos códigos de bom- governo das empresas cotadas na Colômbia}

Resumo No presente artigo é estudada a revelação de diretrizes sobre responsabilidade social nos códigos de bom-governo das empresas listadas no mercado público de valores da Colômbia. Para isso, identificaram-se os códigos de bom-governo disponíveis nos sites das empresas que conformam a cesta de Índice Geral da Bolsa de Valores da Colômbia, IGBC, vigente para o segundo trimestre de 2013; pesquisaram-se os aspectos próprios das melhores práticas corporativas e aqueles associados com as diretrizes da responsabilidade social empresarial, RSE, em particular, os princípios, a relação com os grupos de interesse, as estratégias e atividades, e a revelação e difusão de informação, entre outros. A partir do exposto é evidente que as empresas analisadas não dão conta da adoção das diretrizes de responsabilidade social por meio de códigos de bom-governo, o que privilegia em maior medida a revelação de informação em temas de estrutura organizacional e financeiros, obrigatórios e necessários para os investidores em ações ou credores financeiros. 
Palavras-chave autor Governo corporativo; empresas cotadas; responsabilidade social; revelação e divulgação da informação

\section{Introducción}

Desde sus inicios, la empresa ha sido concebida como un ente productor de bienes y servicios, creada con el fin de satisfacer necesidades específicas de la sociedad en la que se desenvuelve y cuyo objetivo principal, visto desde esta perspectiva, es proporcionar el máximo beneficio y utilidad a los propietarios.

Sin embargo, este enfoque puramente capitalista ha ido evolucionando y modificándose con el transcurrir de los años, como consecuencia, entre otras cosas, de la crisis de confianza vivida a principios del presente siglo en países como Estados Unidos, Italia y Reino Unido, durante la cual se evidenciaron situaciones no deseadas de fraude empresarial, producto de la ambición por parte de los administradores e inversionistas, la ineficacia de la regulación e intervención por parte de los organismos del Estado, y el incumplimiento de los códigos de buen gobierno y de conducta corporativa.

De igual forma, el desarrollo de actividades de las organizaciones ha traído consigo impactos negativos en la sociedad y en el medio ambiente, representados en situaciones de desigualdad laboral, violación de los derechos humanos y deterioro de los recursos naturales, entre otros.

Este panorama ha conllevado problemas de pobreza, desigualdad y cambio climático, que no pueden ser desconocidos por las organiza- ciones, dado su papel frente a estos y la responsabilidad que deben asumir en temas asociados con lo económico, social y ambiental.

Por lo anterior, la sociedad espera que los entes económicos trasciendan de ser generadores de riquezas individuales y lleguen a conjugar sus intereses particulares con los de su entorno, es decir, identifiquen y trabajen en el marco de las relaciones con sus grupos de interés.

Esta postura ha sido acogida a partir de considerar un desarrollo sostenible para el mundo, cuya premisa principal es: "Satisfacer las necesidades del presente sin poner en peligro la capacidad de las generaciones futuras, para satisfacer sus propias necesidades" (Global Reporting Initiative, GRI, 2006).

En este sentido, se han configurado iniciativas en el ámbito mundial, como la desarrollada por la Organización de Naciones Unidas en el Pacto Global, que busca que las empresas y las instituciones del Estado, entre otros tipos de organizaciones, se comprometan a trabajar en pro de la sociedad, siguiendo los principios y lineamientos en materia de cuidado del medio ambiente, protección de los derechos humanos, condiciones laborales y prácticas anticorrupción (United Nations Global Compact, 2012).

Con estos parámetros, las empresas deben materializar y hacer visibles las políticas, prácticas, procesos y resultados, que se originan de la adopción de lineamientos, para dar cuenta de una gestión social y ambientalmente responsable.

En este sentido, los grupos de interés demandan la revelación de información que les permita conocer de manera amplia e integral las cuestiones asociadas con la responsabilidad 
social empresarial de las compañías, RSE. Esta información incluye los códigos de buen gobierno de las empresas, ya que allí se plantean las reglas y los lineamientos que determinan la manera de gobernar las organizaciones, y se espera que en ellos el ente económico declare el compromiso y la responsabilidad ante sus grupos de interés.

Este contexto no es ajeno para el caso colombiano y, por ello, surge la necesidad de evidenciar las prácticas en materia de revelación de información sobre responsabilidad social por parte de las empresas colombianas. En el caso particular del estudio, se propone el análisis a partir de la indagación de los códigos de buen gobierno que publican las empresas que cotizan en el mercado público de valores de Colombia, en sus sitios web. Se selecciona este conjunto de empresas al considerar que son diversas en sus características, como participación significativa de mercado, vinculación a distintos sectores económicos y el impacto social, económico y ambiental que pueden generar sus actividades, por lo cual son empresas de las que se espera adopten y den cuenta de la aplicación de prácticas de responsabilidad social y de buen gobierno.

El conjunto de empresas que se abordan en el presente estudio tiene la obligación de preparar códigos de buen gobierno y de hacerlos accesibles en sus sitios web a las partes interesadas, como lo demanda el código de buenas prácticas empresariales definido en el Anexo 2 de las circulares externas 028 de 2007 y 007 de 2011, emitidas por la Superintendencia Financiera de Colombia. A partir de estos códigos, se espera que las empresas declaren y den a conocer los lineamientos en materia de responsabilidad social que siguen y que deberían acompañar su quehacer empresarial.

Para cumplir lo anterior, el artículo aborda los referentes teóricos y conceptuales, y los lineamientos que se han desarrollado en materia de responsabilidad social y gobierno corporativo, para proponer posteriormente la metodología para desarrollar el trabajo empírico que se demanda en el estudio y, por último, presentar los resultados y las conclusiones de la investigación.

\section{La responsabilidad social y los códigos de buen gobierno en las empresas}

La responsabilidad social empresarial, RSE, al ser considerada un área de estudio ha sido definida, analizada e interpretada de diferentes maneras por varias instituciones, autores, especialistas y empresarios; lo que hace que su concepto y enfoque hayan evolucionado, pues según José Vásquez y Diana Gonzales (2009), con base en Jean Pasquero (2000), la RSE entre 1880 y 1920 era inducida; durante la década de 1930, era enmarcada; entre 1960 y 1980, obligatoria y, luego en los años 1980, la RSE se reconocía como voluntaria (pp. 3-4). Esta última postura es la acogida por la Asociación Española de Contabilidad y Administración de Empresas, AECA (2003), al señalar que: "la RSE es un compromiso voluntario de las empresas con el desarrollo de la sociedad y la preservación del medio ambiente, desde su composición social y un comportamiento responsable hacia las per- 
sonas y grupos sociales con quienes se interactúa” (p. 9).

No obstante, en 2011, la Comisión Europea señaló que la RSE es "la Responsabilidad de las empresas por su impacto en la sociedad" (p. 7), lo cual puede llevar a que la RSE se configure como una obligación implícita de las organizaciones, que trasciende el escenario de las obligaciones legales y, por ende, se configura en un acto consciente y libre de reconocer las consecuencias de los impactos sociales y ambientales que generan las actividades empresariales que estas desarrollan.

Por otra parte, Álvaro Hamburger (2008) propone la RSE como: "El reconocimiento y la aceptación, por parte de la empresa, de los efectos que su actividad produce en el entorno social, considerando este desde tres dimensiones: lo humano, lo ecológico y lo comunitario" (p. 315).

Como es de anotar, la idea principal de esta definición es aceptar y reconocer el efecto que pueda causar la organización en el entorno en el cual ejerce sus actividades diarias; este punto es relevante porque se crea un vínculo directo con la comunidad, y se entiende que los resultados de la organización no son exclusivamente económicos de corto plazo, sino que incorporan resultados ambientales y sociales, que son de interés del entorno en el cual opera.

Además, el Banco Interamericano de Desarrollo, BID (2003), sostiene que:

La RSE es un enfoque que se basa en un conjunto integral de políticas, prácticas y programas centrados en el respeto por la ética, las personas, las comunidades y el medio ambiente. Esta estrategia aplicada a la toma de decisiones y las operaciones de las empresas aumenta el valor agregado y de esa manera mejora su competitividad.

A partir de esta definición, se entiende que la RSE se materializa en un conjunto de lineamientos que llevan al desarrollo de prácticas y programas, que buscan reconocer al otro, y que en el ámbito empresarial están representados por personas que están dentro o fuera de la organización. Los empleados, directivos y propietarios hacen parte del ámbito interno; y el externo está conformado por clientes, proveedores, competidores, gobierno, agentes sociales y comunidad local, entre otros.

A partir de estos diferentes puntos de vista y enfoques que se le han dado a la RSE, es importante resaltar tres aspectos comunes y relevantes que surgen de la interpretación de estas definiciones. En primer lugar, está la relación que debe adoptar la organización con sus grupos de interés o stakeholders ${ }^{1}$. Estos actores son los receptores directos del comportamiento socialmente responsable de la empresa; por ello, se deben identificar y atender los intereses legítimos y necesidades que se generen en cada uno de ellos, como consecuencia de los impactos generados en el desarrollo de la actividad empresarial; por ejemplo, satisfaciendo la necesidad de información, posibilitando

\footnotetext{
1 Los grupos de interés o stakeholders están definidos como: "todo grupo social e individuos afectados de una u otra forma por la existencia de la empresa, con un interés legítimo directo o indirecto por la marcha de esta, que influyen a su vez en la contribución de los objetivos marcados y su supervivencia" (Asociación Española de Contabilidad y Administración de Empresas, AECA, 2003).
} 
la participación en la toma de decisiones que afecten el entorno y generando beneficio mutuo, lo cual lleva a restablecer o consolidar la credibilidad y la confianza ante los grupos de interés.

En segundo lugar, se tiene el reconocimiento del impacto económico, social y ambiental de las actividades empresariales. A partir de este reconocimiento, la organización puede identificar y adoptar prácticas que permitan mitigar el impacto en esta triple dimensión, teniendo como objetivo principal la consecución del desarrollo sostenible y la generación de valor para todos los grupos de interés en el largo plazo.

Por último, se hace necesario considerar un tercer aspecto relevante: la configuración de lineamientos, políticas y programas dentro de las organizaciones que enmarquen su quehacer en términos de su RSE; con ello, se materializa y se manifiestan sus actos ante la responsabilidad asumida con la sociedad y el medio ambiente.

Los lineamientos, políticas y programas definidos deben ser conocidos por los grupos de interés, para que sean entendidos, asumidos y conocidos por los integrantes y los grupos externos a la organización. Las empresas deben revelar la información que resulta relevante para la toma de decisiones en materia económica, social y ambiental, y divulgarla en los medios de comunicación que permitan el mayor acceso y disposición de ella.

Con lo anterior, se desarrolla un elemento determinante de la RSE: la transparencia, pues como señala AECA (2003, pp. 20-21):

Es la pieza básica de la responsabilidad social corporativa. Está basada en el acceso a la información que la organización proporciona sobre su comportamiento social y que es permeable a las expectativas sociales... las organizaciones poco transparentes no asumen el concepto ni los objetivos de la responsabilidad social corporativa. La relación con los grupos de interés obliga a ser transparentes respecto al comportamiento de la organización... un instrumento esencial de la transparencia es la comunicación de los aspectos ligados a la responsabilidad social corporativa mediante un informe dirigido a sus grupos de interés en el que se refleje el compromiso y la participación de los mismos.

Por lo anterior, la información que se revela y divulga al respecto es variada; resalta aquella que se expone en los códigos de buen gobierno, las memorias de sostenibilidad o los informes de responsabilidad social, y en los informes producto de las certificaciones de calidad de la gestión medioambiental o de RSE como ISO 14001 e ISO 26000, entre otros.

De este conjunto, se identifica que el documento base y en el que se podrían configurar las directrices y las reglas de gobierno y de actuación organizacional frente a la RSE, es el código de buen gobierno, ya que desde este se deduce la forma en que la organización identifica las relaciones con sus grupos de interés, plantea sus principios, establece procesos de toma de decisiones y resolución de conflictos de interés que tienen impacto en lo económico, social y ambiental, y se reconoce el alcance de la revelación de la información financiera y no financiera de la empresa. 
Las empresas pueden configurar sus lineamientos asociados con la RSE, desde lo planteado en sus memorias de sostenibilidad o informes de responsabilidad social; no obstante, al observar lineamientos sobre los cuales se elaboran estos informes, como la Guía de elaboración de memorias de responsabilidad social, de la Global Reporting Initiative (GRI, 2006, 2013) y al definir el contenido de la memoria, se debe describir el perfil de la organización. Para ello, se debe considerar lo asociado con el gobierno corporativo, compromisos, retos y participación de grupos de interés, lo cual tendría que ser la expresión de lo señalado por las empresas en sus códigos y prácticas de gobierno corporativo, al tratar aspectos relacionados con sus comités, la estructura de gobierno, roles y remuneración de sus miembros, entre otros. Por ello, los códigos de buen gobierno son el primer instrumento de definición de los lineamientos y políticas de RSE, y posteriormente se configuran en un documento fuente para la elaboración de los informes de responsabilidad social.

Los códigos de buen gobierno se soportan en la concepción de Gobierno Corporativo, GC que, según la Organización para la Cooperación y el Desarrollo Económicos² (OCDE, 2004, p. 11), se define como:

Una serie de relaciones entre el cuerpo directivo de una empresa, su consejo, sus accionistas y otras partes interesadas. El go-

2 Esta organización es el principal referente internacional, ya que ha emitido principios o recomendaciones reconocidos por la mayoría de los países, y que han enmarcado los lineamientos que se siguen en materia de gobierno corporativo.

\begin{abstract}
bierno corporativo también proporciona una estructura para el establecimiento de objetivos por parte de la empresa, y determina los medios que pueden utilizarse para alcanzar dichos objetivos y para supervisar su cumplimiento.
\end{abstract}

Dicho esto, la importancia de los códigos de buen gobierno radica en que el GC pretende asegurar un adecuado manejo en la administración, mejorar su funcionamiento interno y externo, a partir del cumplimiento de responsabilidades, y asegurar un comportamiento ético organizacional basado en estructuras que permitan tomar decisiones adecuadas.

De igual forma, la institución antes citada configura los principios que enmarcan al gobierno corporativo:

a. Asegurar las bases para un marco efectivo de GC que promueva los mercados eficientes y transparentes, consistentes con la ley y señalando claramente la división de responsabilidades entre los auditores encargados de la supervisión, regulación e implementación de normas relacionadas con el GC.

b. Proteger y facilitar el ejercicio de los derechos de los accionistas, así como las funciones claves que poseen como dueños.

c. Asegurar un tratamiento equitativo de todos los accionistas, incluyendo los minoritarios y extranjeros, ya que estos pudieran tener una compensación por la violación de sus derechos.

d. Reconocer los derechos de los stakeholders en la información de la entidad, establecidos por la ley o por medio de acuerdo 
mutuo; así como fomentar la cooperación activa entre corporación y stakeholders en el desarrollo de la salud, empleos y el sostenimiento de las empresas financieramente.

e. Asegurar un oportuno y acertado acceso - y transparencia- a la información de la entidad, incluyendo su situación financiera, desempeño, directorio y gobierno.

f. Asegurar una guía estratégica dentro de la compañía, el efectivo monitoreo del consejo de administración y sus responsabilidades.

Estos principios incrementan el reconocimiento de las relaciones de la organización con sus grupos de interés, al identificar los derechos de estos últimos y promover la cooperación en el desarrollo de temas como la salud y el empleo de las personas que están en su entorno y el sostenimiento de las empresas, entre otros aspectos, lo que lleva al compromiso que debe tener la empresa con estos y se traduce en un escenario de fomento a la responsabilidad social de la empresa.

Por lo anterior, se esperaría que en los códigos de buen gobierno se declaren los aspectos asociados con la RSE que harían parte de este o que se requeriría de su configuración para poder definir los aspectos asociados con tal responsabilidad. Así mismo, la revelación y divulgación de esta información a sus grupos de interés es determinante para consolidar el compromiso con la transparencia que demanda este tema, de manera que contribuya a la confianza en las organizaciones, lo que es valorado por los distintos grupos de interés que desean conocer en todo momento determinados aspectos esen- ciales, y el impacto presente y futuro de la organización (AECA, 2003).

\section{Antecedentes de investigación}

La responsabilidad social, los lineamientos de gobierno corporativo y la revelación de información asociada a estos temas se han configurado como prácticas que las empresas han adoptado de manera importante desde finales de los años noventa, trayendo consigo beneficios económicos, organizacionales y reputacionales tanto para estas como para el entorno en el que se desarrollan (International Finance Corporation, IFC, 2005).

Esta evolución en la revelación de información asociada a la RSE y vinculada a los lineamientos de gobierno de las empresas, tiene sustento en la búsqueda de una mejora en la transparencia informativa, lo que lleva a la reducción de asimetrías de la información y los costos de agencia, como lo exponen Isabel García, Luis Rodríguez e Isabel Gallego (2011) en un estudio previo sobre gobierno corporativo e información estratégica en internet. Esta relación entre el gobierno corporativo y la RSE se define a partir del grado de efectividad del gobierno de las empresas y del reconocimiento de sus grupos de interés; es decir, dadas la amplitud y la rigurosidad en la definición de las prácticas de gobierno corporativo y el vínculo y relaciones que se establezcan con sus grupos de interés, tanto internos como externos, demandará una mayor o menor revelación de información, lo que conlleva no solo agenciar aquella que surja de lineamientos legales y, que normalmente es financiera, sino que se incorpore 
información voluntaria, o no financiera, como la RSE (García, Rodríguez \& Gallego, 2011).

Esta afirmación ha sido ratificada en distintos estudios empíricos, como el desarrollado por José Moneva, Eduardo Ortas e Isabel Acero (2013), que resaltan la evolución que en el ámbito mundial ha tenido el acogimiento de prácticas de responsabilidad social en las entidades, al considerar que la revelación de esta información en las memorias de sostenibilidad con base en los lineamientos de la GRI, ha pasado de 20 entidades que reportaron en 2002 a 1.200 que informaron a finales de 2010.

A partir de este contexto, este mismo estudio realizó una indagación sobre la transparencia y el desempeño social de las cajas de ahorros y las cooperativas de crédito aragonesas (España), considerando, para ello, una lista de indicadores preparada con base en el suplemento del sector de servicios financieros propuesto por la GRI, con los cuales se evaluó el contenido de la información provista en el informe anual, la memoria de obra social, el informe de responsabilidad social y el informe anual de GC.

A partir de lo anterior, se espera que las entidades evaluadas hagan referencia a sus políticas sociales y medioambientales, la estructura de los órganos de gobierno y otros aspectos como la corrupción. Este estudio resulta relevante para la presente investigación en la medida en que reconoce la importancia de declarar los lineamientos asociados con la RSE en los códigos de buen gobierno de las entidades. $\mathrm{Al}$ respecto, el estudio concluye que hay una clara voluntad por parte de las entidades analizadas por el establecimiento de principios y una política social específica. En contraste, la información que se expone no es detallada y es evidente la ausencia en la declaración de los mecanismos de seguimiento y control que desde las memorias de sostenibilidad y de los códigos de buen gobierno se pudieran establecer.

No obstante, esta situación y forma de entender las bondades de la revelación de información asociada con las prácticas de GC y de RSE, parece no ser una generalidad en el ámbito mundial, ya que como lo han demostrado Marcela de Guadalupe Pelayo-Velázquez, Yolanda Fuentes-Callén, Beatriz Cuéllar-Fernández y Myriam Arias-Uribe (2013), en un estudio que aborda el impacto de la divulgación de información en internet de las empresas en América Latina (México, Brasil, Argentina y Chile), en el que se define un índice de divulgación en internet con base en la información contable y financiera, de gobierno corporativo y responsabilidad social e información de contacto, navegabilidad, diseño y accesibilidad, se evidencia que el nivel de divulgación de información financiera y no financiera es menor en los países emergentes que en los países desarrollados. Estos resultados llaman la atención, dado que la importancia de las políticas de transparencia informativa puede contribuir a la reputación de una empresa e influir de manera significativa en su valoración; sin embargo, esta afirmación no es apreciada de la misma manera por las empresas que pertenecen a países de América Latina, como lo expone este estudio.

Otros estudios que ratifican lo anterior son el desarrollado por Fernando Morales-Parada y José Ignacio Jarne-Jarne (2009) y el de María del Pilar Marín-Gaviria, Stella Maldonado- 
García y Laura Sanmiguel-Rodríguez (2012), al reconocer la baja transparencia informativa por parte de las empresas chilenas, que conformaban el IPSA ${ }^{3}$, en asuntos como los códigos de ética, los informes de gobierno corporativo y los informes sociomedioambientales o de gestión, lo cual se detectó a partir de la indagación de la información financiera y no financiera publicada en los sitios web de estas empresas. Así mismo, se concluye que la información divulgada corresponde a los lineamientos de la Global Reporting Initiative, GRI, al hacer uso de los informes anuales y las memorias de responsabilidad social, pero sin aplicar plenamente los indicadores y sin la suficiente profundidad que se requiere al respecto.

En Colombia, resalta el estudio realizado por Manuel Rodríguez y Ana María Justicia (2006), que analizaron el grado de difusión de información financiera en el marco del gobierno corporativo que deberían presentar las principales entidades financieras en sus páginas web. Los resultados evidenciaron un bajo grado de divulgación de prácticas de gobierno corporativo y, sobre aquellas que divulgaron, se reconoce la publicación de información obligatoria de carácter financiero, lo que deja a un lado información de gestión sobre gobierno corporativo y de sostenibilidad.

Por otra parte, Jaime Andrés Correa, Martha Cecilia Álvarez y Andrés Pulgarín (2011) señalan los bajos niveles de divulgación y revelación de la información asociada a la RSE publicada en los sitios web de empresas colom-

3 Índice de Precios Selectivo de Acciones, conformado por las 40 acciones más transadas de la Bolsa de Comercio de Santiago de Chile. bianas que pertenecían al Índice General de la Bolsa de Valores de Colombia, IGBC. Este estudio resalta que para 2009 la revelación de información sobre RSE fue de 33,1\% y para 2010 de 53,2\%. Si bien estos resultados son bajos, muestran una mejoría en la presentación de esta información, debido a que las empresas han reconocido el tema en sus prácticas organizacionales. Así mismo, resalta la utilización de estándares internacionales como los de la GRI, aunque no se hace uso de la totalidad de los indicadores que esta institución plantea.

Este último estudio es cercano a lo previsto en la presente investigación; no obstante, no considera la hipótesis de trabajo de este estudio, al reconocer la revelación de información sobre lineamientos de responsabilidad social en los códigos de gobierno corporativo, es decir, evalúa el contenido de estos últimos en cuanto a la declaración que podrían hacer las empresas con respecto a distintos elementos referentes a la RSE.

En la revisión de la literarura, otro aspecto a considerar es el de las características que puedan tener las entidades que influyen en la revelación de información, como se ha demostrado en investigaciones previas (Católico \& Gómez, 2013; Haro, Alarcón, Caba, 2012; Rodríguez, Gallego \& García, 2010), al considerar que factores como la cotización en un mercado público de valores, el tamaño de la empresa, la concentración sectorial, el tipo de entidad, la aplicación del marco de recomendaciones de la GRI, el sector industrial, y medidas financieras, como la rentabilidad y el endeudamiento, tienen incidencia en este sentido.

De igual forma, el conjunto de estudios abordados deja ver que la información recolectada ha 
sido la reportada en los sitios web, es decir, que si bien se conocen medios de divulgación de la información como prensa, correo físico, boletines y llamadas telefónicas, entre otros, internet es el medio de comunicación que se comienza a privilegiar, dado que permite tener una mayor flexibilidad, disponibilidad, actualización, cobertura y accesibilidad de la información; de esta forma se cumplen eficientemente las necesidades de los grupos de interés y, por supuesto, para la empresa se convierte en una ventaja revelar la información al menor costo posible, como señalan María del Mar Gálvez-Rodríguez, María del Carmen Caba-Pérez y Manuel López-Godoy (2012); Fernando Morales-Parada y José Ignacio Jarne-Jarne (2009); y Fabio Gómez y Diego Católico (2009).

Por último, las investigaciones previas muestran que para evaluar la revelación de información asociada a la RSE y gobierno corporativo, se hace uso de índices, comparaciones anuales, análisis de contenido de los informes, para poder acopiar y analizar la información revelada por parte de las distintas organizaciones en esta materia; es decir, los estudios reconocen la aplicación de métodos e instrumentos para poder trascender los trabajos empíricos, al abordar distintos escenarios y distintos tipos de organizaciones, con lo cual se ratifica su importancia en el desarrollo de las organizaciones en el ámbito internacional y nacional.

\section{Diseño de la investigación}

La presente investigación es empírico-descriptiva, dado que busca determinar el grado de revelación de lineamientos sobre responsabili- dad social en los códigos de buen gobierno publicados en los sitios web de las empresas que cotizan en el mercado público de valores de Colombia.

Para ello, como objeto de estudio, se seleccionaron las empresas cotizadas pertenecientes a la canasta 48 del Índice General de la Bolsa de Valores de Colombia (IGBC) ${ }^{4}$, vigente para el segundo trimestre de 2013 (Bolsa de Valores de Colombia, 2013), como lo han estimado en estudios previos Jaime Andrés Correa, Martha Cecilia Álvarez y Andrés Pulgarín (2011); Fernando Morales-Parada y José Ignacio JarneJarne (2009); y María del Pilar Marín-Gaviria, Stella Maldonado-García y Laura Sanmiguel-Rodríguez (2012). Estas empresas tienen distintas características que motivan la consideración de incorporarlas en un estudio como el que se desarrolla, al identificar que son de interés público, pertenecientes a distintos sectores económicos y cuyas actividades generan un impacto social, económico y ambiental significativo, por lo cual son empresas de las que se espera adopten y den cuenta de la aplicación de prácticas de responsabilidad social y de buen gobierno, vinculando lo primero a la hora de configurar lo segundo. En total, se trabaja con una canasta compuesta por 37 títulos valores representados en 32 empresas, pues la canasta 48 está compuesta por las acciones más representativas del mercado de renta variable.

El estudio no trata de establecer si estas características son determinantes de la revelación de la información sobre responsabilidad

4 Este índice bursátil permite evaluar la evolución y tendencia del mercado accionario, al dar información sobre qué acciones son más rentables en el país (IGBC, 2013). 
social en los códigos de buen gobierno, como lo han realizado previamente Luis RodríguezDomínguez, Isabel Gallego-Álvarez e Isabel María García-Sánchez (2010), sino que la consideración se centra en entender que se está ante un conjunto de empresas que recogen distintos aspectos de la estructura empresarial colombiana y, por tanto, puede lograrse una aproximación al comportamiento general de las empresas.

Con lo enunciado por la Superintendencia Financiera de Colombia en sus circulares 028 de 2007 y 007 de 2011, como guía de las mejores prácticas corporativas que se espera sean adoptadas y llevadas a cabo por las sociedades emisoras de valores que pretendan evaluar sus estándares de gobierno corporativo, en materia de asamblea general de accionistas, junta directiva, revelación de información financiera y no financiera y solución de controversias, se diseñó una matriz con elementos propios de gobierno corporativo, incluyendo aspectos referentes a la responsabilidad social empresarial, de manera que se pueda analizar la revelación de información sobre lineamientos de RSE en los códigos de buen gobierno corporativo.

A partir de lo anterior, se configuraron 11 categorías para las empresas objeto de estudio, así: 1) principios orientadores, 2) observancia de lineamientos sobre GC y RSE, 3) identificación de las relaciones con los grupos de interés, 4) máximo órgano social y derechos de los accionistas, 5) estructura organizacional, 6) junta o consejo directivo, 7) administradores, 8) órganos de control, 9) revelación de la información, 10) divulgación de la información (facilidad de acceso a la información), y 11) autorregulación del gobierno corporativo.

Los aspectos que comprenden cada una de estas categorías se identifican como variables, las cuales han sido planteadas a partir de lo expuesto en el marco teórico en cuanto a la lógica que enmarca la RSE a partir de sus principios, la relación con los grupos de interés, las dimensiones económica, social y ambiental que la constituyen, entre otros, que así mismo, son variables abordadas en estudios empíricos como los desarrollados por José Moneva, Eduardo Ortas e Isabel Acero (2013); Marcela de Guadalupe Pelayo-Velázquez, Yolanda Fuentes-Callén, Beatriz Cuéllar-Fernández y Myriam Arias-Uribe (2013), y definidas en lineamientos internacionales como la ISO 26000 y la Guía para la elaboración de memorias de sostenibilidad, emitida por la GRI en 2013, versión 4.0.

El conjunto de variables que comprende cada una de las 11 categorías expuestas son evaluadas con una puntuación individual de 1 o 0 , es decir, se trabajan como variables dicotómicas (Terence E. Cooke, 1989; Marcela de Guadalupe Pelayo-Velázquez, Yolanda Fuentes-Callén, Beatriz Cuéllar-Fernández y Myriam Arias-Uribe, 2013; Diego Católico \& Fabio Gómez, 2013), obteniendo uno (1) cuando la empresa informa sobre el ítem a evaluar y cero ( 0 ) cuando no da cuenta al respecto.

Los datos recolectados con relación a las categorías y variables definidas, se obtienen de la revisión de los códigos de buen gobierno publicados en los sitios web de las empresas que constituyen el IGBC, es decir, se realiza un análisis de contenido de aquella información que 
resulta ser de fácil acceso para todos y cada uno de los grupos de interés, de manera que allí se espera conocer los lineamientos establecidos por las organizaciones a la hora de configurar sus reglas, políticas y prácticas en términos de su gobierno organizacional.

Los códigos de buen gobierno que declaran las empresas como vigentes tienen como fecha de expedición, en la mayoría de los casos, los años 2011 o 2012, lo que responde a las más recientes versiones emitidas con ocasión a los ajustes realizados a propósito de lo definido en la circular 007 de 2011.

A partir de lo anterior, se realizó una verificación sobre las categorías y variables configuradas en todas las empresas objeto de estudio, con lo cual se obtiene una puntuación por categoría a partir de las evaluaciones obtenidas en las variables que las constituyen; con ello, se analiza el grado de revelación de la información en cada una de las categorías que dan cuenta de los aspectos relevantes de los códigos de buen gobierno, asociados con los elementos propios de la responsabilidad social empresarial. Para ello, se considera la siguiente expresión matemática que formaliza la manera de llegar al grado de revelación para cada categoría y variable:

$$
\text { Grado de revelación }=\sum X_{i} / n_{i=1}^{n}
$$

\section{Donde}

$\mathrm{X}$ corresponde a cada categoría y/o variable punteada con 1 o 0 , y las $\mathrm{X}_{\mathrm{i}}$ consideran un máximo de 31 observaciones, dado que $n$ es el número de empresas evaluadas.
Del total de las treinta y dos (32) empresas que conforman el presente estudio, la empresa Biomax Biocombustibles S.A. no tenía disponible el código de buen gobierno en su página web para la fecha que se realizó la indagación y, por ende, no se incorporó en los resultados del estudio, lo que significa que la máxima puntuación obtenida por cada categoría y variable es de 31 puntos.

\section{Resultados de la investigación}

Una vez realizada la indagación de los datos de las empresas seleccionadas, se presentan los resultados obtenidos para cada categoría.

En términos generales, ninguna categoría y variable obtiene la máxima puntuación (31 puntos), por lo que las empresas no logran resultados deseados a la hora de exponer la información concerniente al gobierno corporativo y a los aspectos propios de la responsabilidad social que se proponen sean contemplados en estos códigos, es decir, si bien las empresas cumplen la definición de un código de buen gobierno, las empresas analizadas no declaran en este los aspectos asociados a la RSE. Esto significa que las empresas no consideran este tema como un elemento que incorpora una obligación implícita y, por ende, desde sus códigos de buen gobierno, las empresas no consideran políticas, ni lineamientos para gestionar el impacto social y ambiental que se origina como producto del desarrollo de sus actividades empresariales.

Al detallar los resultados, la primera categoría analizada arroja las siguientes puntuaciones. 


\begin{tabular}{|c|c|}
\hline Categoría y variables & $\begin{array}{l}\text { Revelación de la } \\
\text { información (puntaje) }\end{array}$ \\
\hline Definición de principios orientadores de gobierno corporativo & 15 \\
\hline $\begin{array}{l}\text { * Configuración de principios orientadores de la RSE en el marco del gobierno } \\
\text { corporativo: }\end{array}$ & 3 \\
\hline - Rendición de cuentas & 0 \\
\hline - Transparencia & 3 \\
\hline - Comportamiento ético & 2 \\
\hline - Respeto por los intereses de las partes interesadas & 0 \\
\hline - Respeto por la ley & 2 \\
\hline - Respeto por la normatividad internacional de comportamiento & 0 \\
\hline - Otros & 1 \\
\hline
\end{tabular}

Tabla 1. Definición de los principios orientadores de buen gobierno enfocados hacia la RSE Fuente: elaboración propia

De acuerdo con las definiciones realizadas en la ISO 26000 sobre la necesidad de definir los principios que deben orientar la responsabilidad social en las organizaciones, la mitad de las empresas objeto de análisis (15/31), que representan el 48\%, define principios orientadores de gobierno corporativo al momento de configurar sus códigos en esa materia. De estas empresas, solo tres (3) declaran principios asociados con la responsabilidad social; de manera particular, se trata lo referido a la transparencia en tres (3) empresas, comportamiento ético en dos (2) empresas y respeto por la ley en dos (2) empresas. El principio de transparencia es común en los casos analizados cuando se trata de declarar principios organizacionales que den cuentan de sus prácticas de RSE.

Siguiendo lo definido por AECA (2003) y comentado en el marco teórico sobre la importancia de la transparencia en la RSE, estas em- presas fortalecen sus relaciones con los grupos de interés y conciben el hecho de posibilitar el acceso a la información para lograr una mayor aceptación y beneplácito ante sus stakeholders como un elemento fundamental.

No obstante, la mayoría de las empresas analizadas considera como principios aquellos que van orientados a las necesidades de regulación en cuanto al trato equitativo con los accionistas y aquellos otros aspectos financieros que son requeridos desde lineamientos como el emitido por la Superintendencia Financiera de Colombia (2011), lo que deja de lado elementos esenciales que podrían contribuir al desarrollo de prácticas en las organizaciones que atiendan su responsabilidad ante los impactos sociales y ambientales que generan sus actividades empresariales.

Esta situación se complementa con lo observado frente a los lineamientos que las empresas declaran seguir para configurar sus códigos de buen gobierno, como se expone a continuación. 
REVELACIÓN DE LA RSE EN Códigos de BUEN GOBIERNo / L. ROMERo, L. WANUMEN, D. CATólico / 613

\begin{tabular}{|c|c|}
\hline Categoría y variables & $\begin{array}{c}\text { Revelación de la } \\
\text { información (puntaje) }\end{array}$ \\
\hline Observancia de lineamientos sobre GC y RSE & 13 \\
\hline * Lineamientos internacionales sobre gobierno corporativo & 2 \\
\hline - OCDE & 1 \\
\hline - Corporación Andina de Fomento, CAF & 1 \\
\hline - Otros & 0 \\
\hline * Lineamientos propios nacionales sobre gobierno corporativo & 11 \\
\hline - Superintendencia Financiera & 7 \\
\hline - Superintendencia de Sociedades y Confecámaras & 0 \\
\hline - Otros & 4 \\
\hline * Lineamientos internacionales sobre responsabilidad social & 2 \\
\hline - ISO 14001 (gestión medioambiental) & 2 \\
\hline - ISO 26000 (responsabilidad social) & 0 \\
\hline - Global Reporting Initiative (Guía para las memorias de sostenibilidad) & 0 \\
\hline $\begin{array}{l}\text { - Pacto Global (principios universalmente aceptados en derechos humanos, } \\
\text { estándares laborales, medioambiente y anticorrupción) }\end{array}$ & 0 \\
\hline - Otros & 0 \\
\hline * Lineamientos propios nacionales sobre responsabilidad social & 1 \\
\hline - Otros & 1 \\
\hline
\end{tabular}

Tabla 2. Definición de políticas a partir de la observancia de lineamientos y guías nacionales e internacionales en materia de GC y RSE

Fuente: elaboración propia

En lo que se refiere a la observancia de lineamientos internacionales y nacionales sobre gobierno corporativo y/o responsabilidad social, se reconoce que el $42 \%$ (13/31) de las empresas declara en su código de buen gobierno acogerse a alguno de los lineamientos que se tienen al respecto. De los lineamientos que se observan para configurar sus códigos de buen gobierno, once (11) empresas siguen lineamientos locales. La mayoría de estas (7) empresas se soporta en lo establecido por la Superintendencia Financiera de Colombia; es decir, siguen los lineamientos de obligatorio cumplimiento dado el mercado público de valores en el que participan.
A partir de lo anterior, la aplicación de lineamientos que tienen un carácter voluntario, como los referidos a la RSE, son de baja revelación, dado que solo dos empresas declaran aspectos asociados con la ISO 14001, norma que hace referencia a la gestión medioambiental, y ninguna declara adoptar lineamientos como los previstos en la norma ISO 26000 y en la Guía de la GRI. En este sentido, las empresas no logran aplicar una definición de responsabilidad social como la establecida por el Banco Interamericano de Desarrollo (BID, 2007), al señalar que esta responsabilidad es la aplicación voluntaria de políticas, prácticas y 
programas centrados en el respeto por la éti$\mathrm{ca}$, las personas, las comunidades y el medioambiente, es decir, no configuran sobre su forma de gobierno, de manera voluntaria, políticas fundamentales que consideran aspectos propios de la RSE. No obstante, estas empresas pueden seguir otra forma de revelación, como divulgar todo lo concerniente a la RSE en memorias de sostenibilidad, pero como se indicó previamente esta última debe considerar definiciones hechas desde los lineamientos de gobierno corporativo planteados en las empresas; de ahí, la atención que se presta en estos documentos.

Ahora bien, una vez se han considerado los principios y observado lineamientos para definir sus políticas en materia de RSE, otro tema relevante es el de la identificación de las relaciones que la empresa sostiene con sus grupos de interés, las cuales motivan la definición de actividades para mitigar y atender los potenciales impactos que genera el desarrollo de las actividades, y considerar la resolución de conflictos que se presenten con estos.

\begin{tabular}{|c|c|}
\hline Categoría y variables & $\begin{array}{c}\text { Revelación de la } \\
\text { información (puntaje) }\end{array}$ \\
\hline Identificación de las relaciones con sus grupos de interés & 25 \\
\hline * Definición de relación y compromisos con grupos de interés & 24 \\
\hline - Accionistas & 23 \\
\hline - Empleados & 19 \\
\hline - Clientes & 18 \\
\hline - Proveedores & 20 \\
\hline - Acreedores financieros & 4 \\
\hline - Estado & 13 \\
\hline - Comunidad internacional & 0 \\
\hline - Comunidad local & 15 \\
\hline - Otros (1) & 11 \\
\hline * Estrategias y actividades en materia de RSE & 8 \\
\hline * Resolución de conflictos de interés con los grupos de interés & 23 \\
\hline
\end{tabular}

(1) Este ítem hace referencia a grupos como consumidores financieros, junta directiva, alta dirección y competencia, que no son representativos en los resultados obtenidos. Por ello, en este estudio se presentan agregados en esta categoría.

Tabla 3. Identificación de las relaciones con sus grupos de interés Fuente: elaboración propia

En su mayoría (80\%), las empresas reconocen a sus grupos de interés al momento de configurar sus códigos de buen gobierno; este elemento es determinante para pensar que las prácticas de gobierno corporativo dan cuenta de las relaciones de la organiza- ción con esos grupos, como lo plantea AECA (2003).

De las relaciones y compromisos declarados, se resalta su existencia clara y detallada con los accionistas (23 empresas), proveedores (20 empresas), empleados (19 empresas) y clientes (18 
empresas), que conforman la cadena de valor inmediata para este tipo de empresas. De igual forma, en el tema de los accionistas, las empresas revelan un capítulo independiente para reconocer todo lo asociado con ese grupo de interés, para responder a lo establecido por los lineamientos locales, en los cuales se resalta la necesidad de informar en un apartado especial el tema de la asamblea de accionistas (Superintendencia Financiera de Colombia, 2011).

No obstante, de manera voluntaria, ocho empresas (Bancolombia S.A., Construcciones el Cóndor S.A., Ecopetrol S.A., Empresa de Energía de Bogotá S.A. ESP, Enka de Colombia S.A., Grupo de Inversiones Suramericana S.A., Isagen S.A. ESP e Inversiones Argos S.A.) revelan en un capítulo específico aspectos referentes a la RSE, es decir, dan cuenta de las estrategias y actividades a desarrollar con respecto a sus grupos de interés, lo cual da un valor adicional en materia informativa al momento de configurar sus prácticas de buen gobierno ante sus grupos de interés.

En contraste con lo anterior, en un apartado específico, un número importante (23 empresas) examina la resolución de controversias e incorpora a algunos de los grupos de interés que previamente identificó. Este aspecto se deriva de la obligación que existe por la parte de la Superintendencia Financiera de Colombia (2011) de definir este tema, aunque esta se enfoca a los asuntos que se presentan con los accionistas y administradores de las entidades vigiladas.

Así mismo, estos lineamientos en materia de gobierno corporativo exigen la identificación de otros temas, que comprenden cuatro categorías del presente estudio que dan cuenta de la dirección, administración y representación de la sociedad: máximo órgano social y derechos de los accionistas, estructura organizacional, junta o consejo directivo y los administradores.

\begin{tabular}{|c|c|}
\hline Categorías y variables & $\begin{array}{c}\text { Revelación de la } \\
\text { información (puntaje) }\end{array}$ \\
\hline Reconocimiento del máximo órgano social y derechos de los accionistas & 26 \\
\hline * Declaración de posibles conflictos de interés & 21 \\
\hline * Tratamiento equitativo de los accionistas & 24 \\
\hline Consideración de aspectos asociados con la estructura organizacional & 28 \\
\hline * $\quad$ Definición de una estructura organizacional & 28 \\
\hline Definición de políticas y metas concretas para cada órgano o dependencia & 0 \\
\hline $\begin{array}{l}\text { Declaración sobre la responsabilidad frente al cumplimiento de políticas y metas } \\
\text { concretas para cada órgano o dependencia }\end{array}$ & 0 \\
\hline Seguimiento y evaluación de la gestión de los funcionarios responsables & 18 \\
\hline * $\quad$ Adopción e implementación de medidas correctivas & 0 \\
\hline * $\quad$ Reconocimiento del uso de indicadores de gestión & 0 \\
\hline * $\quad$ Exigencia de manuales de funciones y procedimientos & 26 \\
\hline Junta o consejo directivo & 29 \\
\hline * Consideraciones para la toma de decisiones & 13 \\
\hline
\end{tabular}




\begin{tabular}{|c|c|c|}
\hline & Categorías y variables & $\begin{array}{c}\text { Revelación de la } \\
\text { información (puntaje) }\end{array}$ \\
\hline \multirow[t]{8}{*}{ * } & Comités de apoyo para la toma de decisiones & 28 \\
\hline & - Comité de gobierno corporativo & 11 \\
\hline & - Comité de remuneraciones y compensaciones & 3 \\
\hline & - Comité de auditoría & 25 \\
\hline & - Comité de responsabilidad social & 0 \\
\hline & - Comité financiero & 6 \\
\hline & - Otros comités & 17 \\
\hline & - Administradores & 22 \\
\hline * & Criterios sobre perfil profesional y personal & 15 \\
\hline * & Cumplimiento de deberes & 15 \\
\hline * & Exigencia de rendición de cuentas & 6 \\
\hline
\end{tabular}

Tabla 4. Dirección, administración y representación de la sociedad Fuente: elaboración propia

$\mathrm{Al}$ momento de establecer un código de gobierno corporativo, se debe asegurar la existencia de prácticas y mecanismos que permitan una buena gestión del órgano de dirección, con el fin de que las decisiones a tomar se basen en un conjunto de normas y estrategias claras que lleven al logro de los objetivos organizacionales. Los resultados obtenidos permiten evidenciar que las empresas de manera significativa (84\%), reconocen el máximo órgano social y los derechos de los accionistas, pensando en el trato equitativo y el manejo de conflictos de interés que se pueden generar $y$, por ende, deben atender ante este grupo de interés.

Otro punto relevante es el de los resultados obtenidos en los ítems relacionados con la estructura de la organización (90\%). En sus códigos de gobierno, las empresas exponen usualmente la necesidad de definir la estructura organizacional, en términos de los niveles jerárquicos y la exigencia de configurar manuales de funciones y procedimientos. No obstante, las empresas no declaran como requerimiento las políticas y metas concretas para cada órgano y la responsabilidad de estos ante las metas; tampoco reconocen la adopción de medidas correctivas e indicadores de desempeño como políticas para el buen gobierno organizacional.

En cuanto a la junta o consejo directivo, las empresas revelan información asociada con la disposición de tener comités de apoyo para la toma de decisiones (28 empresas). Resalta que el principal comité que se estructura para este fin es el de auditoría (25 empresas). En oposición con lo anterior, ninguna empresa presenta en sus códigos de buen gobierno la constitución de un comité de responsabilidad social, por lo cual no se considera la institucionalidad que debe tener esta responsabilidad en el actuar de las empresas.

Por último, la figura de los administradores cobra sentido en las prácticas de buen gobierno; de allí que las empresas tengan criterios específicos sobre el perfil profesional y personal 
(15 empresas), para dar alcance al cumplimiento de deberes de estas personas. Sin embargo, las empresas no dan una indicación explícita de la exigencia de rendir cuentas de los administradores, pues se debe reconocer que, de acuerdo con la Ley 222 de 1995, en su artículo 45: "los administradores deberán rendir cuentas comprobadas de su gestión al final de cada ejercicio", siendo esto una oportunidad por parte de las empresas de requerir de manera obligada tal acción por parte de estos individuos, para buscar la visibilidad con respecto a la gestión realizada. Esta situación es coincidente con la ausencia de un principio de transparencia, que como se comentó previamente es el primer paso para propiciar la responsabilidad social en las organizaciones.

Dada la ausencia de lo anterior, se requiere la existencia de órganos de control que mitiguen esta situación.

\begin{tabular}{lc}
\hline \multicolumn{1}{c}{ Categorías y variables } & $\begin{array}{c}\text { Revelación de la } \\
\text { información (Puntaje) }\end{array}$ \\
\hline Órganos de control & 27 \\
\hline$* \quad$ Exigencia del control interno & 26 \\
\hline$\cdot$ Revisoría fiscal & 12 \\
\hline$\cdot$ Auditoría interna & 25 \\
\hline$\cdot$ Colaboración con entes de control & 0 \\
\hline$\cdot$ Otros & 9 \\
\hline$*$ Exigencia del control externo & 19 \\
\hline$\cdot$ Revisoría fiscal & 14 \\
\hline$\cdot$ Auditoría especializadas & 8 \\
\hline$\cdot$ Superintendencia Financiera & 13 \\
\hline$\cdot$ Colaboración con entes de control & 9 \\
\hline$\cdot$ Otros & 7 \\
\hline
\end{tabular}

Tabla 5. Definición de órganos de control

Fuente: elaboración propia

El 87\% (27 empresas) declara la definición de órganos de control, en la que la figura de auditor interno es la más reconocida como mecanismo de control interno y la de revisor fiscal como mecanismo externo. Es decir, estos controles tienen un papel importante al verificar que la entidad desarrolle sus actividades de forma adecuada y, por ello, $25 \mathrm{em}$ presas implementan la auditoría interna y 14 contemplan la revisoría fiscal como control externo. No obstante, existen empresas que declaran la figura de la revisoría fiscal como un mecanismo interno de control, aunque no resulta claro el porqué de esta postura ante el control que ejerce este ejercicio profesional.

Así mismo, la exigencia de revelar y divulgar la información contribuye a la lógica de rendición de cuentas y la búsqueda de la transparencia en las organizaciones. Al respecto, se obtuvieron los siguientes resultados. 


\begin{tabular}{lc}
\hline \multicolumn{1}{c}{ Categorías y variables } & $\begin{array}{c}\text { Revelación de la } \\
\text { información (puntaje) }\end{array}$ \\
\hline Revelación de información & 22 \\
\hline$* \quad$ Revelación de información y controles financieros & 22 \\
\hline$\cdot$ Estados financieros & 21 \\
\hline • Informe de revisoría fiscal o auditoría & 15 \\
\hline - Otra & 15 \\
\hline Revelación de información y controles no financieros & 5 \\
\hline - Social & 0 \\
\hline - Ambiental & 0 \\
\hline - Otra & 5 \\
\hline sitios web) & 19 \\
\hline
\end{tabular}

Tabla 6. Revelación y divulgación de la información financiera y no financiera Fuente: elaboración propia

Como se evidencia, el 71\% de las empresas (22/31) enuncia la revelación de información como elemento sustancial en la configuración de sus códigos de buen gobierno. La información asociada con aspectos financieros logra porcentajes significativos, lo que muestra que las organizaciones están cumpliendo este elemento, al incrementar la transparencia de las entidades ante sus grupos de interés, hacer énfasis en la publicación de sus estados financieros y reconocer, en ciertos casos, los informes de control como un complemento.

Sin embargo, la exigencia de la revelación de información no financiera es poco declarada, es decir, aquella información que se define a partir de los aspectos propios de la responsabilidad social y que se esperaría las empresas expusieran sugiriendo el uso de memorias de sostenibilidad o informes de responsabilidad social, aunque cinco casos de la muestra hacen referencia a la necesidad de publicar información con respecto a estrategias de la sociedad, investigaciones administrativas, procesos judiciales, cambios en la imagen corporativa, principales proyectos, derechos y procedimientos de votación, entre otros temas.

De igual forma, en el $61 \%$ de los casos, en los códigos de buen gobierno se expresa que los sitios web corporativos son la herramienta idónea para posibilitar el acceso y la oportunidad de la información y para facilitar la comunicación entre la empresa y los grupos de interés, pues permiten el acceso a una mayor información para entender la gestión que la entidad desarrolla.

Por último, se destaca la existencia de un elemento que debe quedar expreso en los códigos corporativos: el asociado con la autorregulación en esta materia, pues el 35\% de las empresas plantea la documentación o generación de informes sobre el cumplimiento del código de buen gobierno y el 32\% hace la exposición de un código de ética o de conducta que oriente el quehacer y las buenas 
prácticas por parte del gobierno corporativo y los integrantes que orientan y constituyen las organizaciones, como se muestra en la tabla 7 .
A partir de las evaluaciones realizadas, se presenta un cuadro que agrega cada una de las categorías analizadas y se observa el grado de revelación para cada una de ellas.

\begin{tabular}{lc}
\multicolumn{1}{c}{ Categoría y variables } & $\begin{array}{c}\text { Revelación de la } \\
\text { información (puntaje) }\end{array}$ \\
\hline Autorregulación del gobierno corporativo & 15 \\
\hline Documentación de las prácticas de buen gobierno & 11 \\
\hline Línea ética o código de conducta & 10 \\
\hline
\end{tabular}

Tabla 7. Autorregulación del gobierno corporativo

Fuente: elaboración propia

\begin{tabular}{lcc}
\hline & \multicolumn{1}{c}{ Categoría de análisis } & $\begin{array}{c}\text { Grado de revelación de } \\
\text { la información }\end{array}$ \\
\cline { 2 - 3 } & $\begin{array}{c}\text { Valor } \\
\text { absoluto }\end{array}$ & $\begin{array}{c}\text { Valor } \\
\text { relativo (\%) }\end{array}$ \\
\hline Definición de principios orientadores de gobierno corporativo & 15 & 48,4 \\
\hline Observancia de lineamientos sobre GC y RSE & 13 & 41,9 \\
\hline Identificación de las relaciones con sus grupos de interés & 25 & 80,6 \\
\hline Reconocimiento del máximo órgano social y derechos de los accionistas & 26 & 83,9 \\
\hline Consideración de aspectos asociados con la estructura organizacional & 28 & 90,3 \\
\hline Junta o consejo directivo & 29 & 93,5 \\
\hline Administradores & 22 & 70,9 \\
\hline Órganos de control & 27 & 87,1 \\
\hline Revelación de información & 22 & 70,9 \\
\hline Divulgación de la información (facilidad de acceso a la información en sitios web) & 19 & 61,3 \\
\hline Autorregulación del gobierno corporativo & 15 & 48,4 \\
\hline
\end{tabular}

Tabla 8. Grado de revelación de información en cada categoría evaluada

Fuente: elaboración propia

Como se observa en la tabla, los porcentajes obtenidos son importantes en términos del grado de revelación en cada categoría; no obstante, esto se debe a que estos resultados son el reflejo del cumplimiento de aquellos aspectos explícitos en los lineamientos dados por la Superintendencia Financiera de Colombia o por las prácticas de gobierno corporativo en el 
ámbito internacional, para los cuales los accionistas son el grupo de interés principal y desde allí se plantean los aspectos definidos en sus códigos, pero cuando se trata de considerar a los demás grupos de interés y, por ende, reflejar sus actuaciones en materia de RSE, los resultados son muy distintos, como se mencionó al analizar cada una de las categorías. Por esto se concluye que el estudio logra los mismos resultados obtenidos previamente por Marcela Pelayo, Yolanda Fuentes, Beatriz Cuéllar y Myriam Arias (2013); José Moneva, Eduardo Ortas e Isabel Acero (2013); Manuel Pedro RodríguezBolívar y Ana María Carolina Justicia-Jiménez (2006), que resaltan que las empresas aún deben hacer esfuerzos importantes por revelar la información asociada con la RSE y vincularla con los aspectos de gobierno corporativo.

\section{Conclusiones}

En el marco de la responsabilidad social empresarial, las empresas no deben ver los lineamientos que se han configurado al respecto $y$, así mismo, los previstos en materia de gobierno corporativo, como un simple proceso normativo. Estos lineamientos son la prueba de la firme intención de las empresas de demostrar a sus grupos de interés, que son transparentes, eficaces, eficientes y ante todo que tienen un alto sentido de compromiso ante lo social, económico y medioambiental.

En este sentido, al configurar sus políticas, estrategias y prácticas de gobierno, las empresas deberían considerar aspectos asociados a la responsabilidad social, con el fin de declarar explícitamente sus intencionalidades como organi- zación ante esta responsabilidad, de tal manera que sus grupos de interés, más allá de sus accionistas, conocieran las pautas en esta materia.

$\mathrm{Al}$ indagar los estudios previos, se observa que las empresas han venido avanzando en la adopción de prácticas tanto en materia de gobierno corporativo como de responsabilidad social; no obstante, cuando se trata de evaluar la transparencia y disponibilidad de la información en estos temas, las empresas deben hacer un esfuerzo significativo, sobre todo cuando se ubican en países emergentes, de manera que sus grupos de interés puedan conocer sobre las políticas y el desempeño social y así puedan evaluar el quehacer de estas organizaciones con respecto a la atención que prestan al impacto social y ambiental de sus actividades empresariales.

$\mathrm{Al}$ analizar los datos obtenidos, se encuentra que las empresas cotizadas en Colombia pertenecientes al IGBC no logran los resultados deseados a la hora de declarar la exigencia de principios, lineamientos, estrategias y actividades en materia de responsabilidad social en los códigos de buen gobierno que han configurado. Se observa que estos últimos atienden los principios y lineamientos nacionales e internacionales en materia de gobierno corporativo, que se enfocan en aspectos financieros, y dejan de lado las relaciones con los demás grupos de interés, y por ende, no incorporan orientaciones que traten el impacto de su actividad empresarial en lo social y ambiental.

De igual forma, desde una visión institucional, las empresas no contemplan en su estructura organizacional un comité de responsabilidad social, que promocione, promueva y realice un seguimiento de este tema en la ges- 
tión de sus integrantes. No obstante, algunas empresas comienzan a declarar las estrategias y las prácticas que se requerirían en materia de responsabilidad social en apartados especiales de sus códigos.

Por otra parte, las empresas muestran la exigencia de revelar y divulgar su información, en mayor medida, la referida a los aspectos financieros, y dejan en un segundo plano aquella no financiera, como la que resulta al considerar los aspectos asociados con la RSE. Sin embargo, en los códigos de buen gobierno promueven de manera importante la divulgación de información en sus sitios web, lo que permite un mayor acceso a la información generada por las empresas.

Por último y a partir de los resultados obtenidos, las empresas analizadas tienen una oportunidad de mejorar lo declarado en sus códigos de buen gobierno, al incorporar lineamientos sobre responsabilidad social, lo que traería valor agregado y mejoraría su confiabilidad y transparencia ante los grupos de interés. De igual forma, el regulador, en este caso, la Superintendencia Financiera de Colombia, podría acoger entre sus lineamientos de gobierno corporativo lo señalado por la OCDE en los principios que se declaran en esta materia, en particular, aquel que señala la consideración por parte de las empresas de los intereses de sus stakeholders.

\section{Referencias}

\section{Asociación Española de Contabilidad y}

Administración de Empresas, AECA (2003).

Marco conceptual de la responsabilidad social corporativa. Madrid: Asociación Española de Contabilidad y Administración de Empresas, AECA.
Banco Interamericano de Desarrollo, BID (2003). BID impulsa responsabilidad social empresarial en América Latina. Disponible en: http://www.iadb.org/es/noticias/ comunicados-de-prensa/2003-08-04/bidimpulsa-responsabilidad-social-empresarialen-america-latina,413.html

Bolsa de Valores de Colombia, BVC (2013). Índice general de la bolsa de valores. Disponible en: http://www.bvc.com.co Católico, Diego \& Gómez, Fabio (2013). Transparencia informativa on-line en empresas colombianas. Revista Internacional Legis de Contabilidad \& Auditoría, 55, 93-118. Colombia (1995). Ley 222 de 1995, por la cual se modifica el Libro II del Código de Comercio, se expide un nuevo régimen de procesos concursales y se dictan otras disposiciones. Diario Oficial, 42.156, 20 de diciembre de 1995. Disponible en: http:// www.alcaldiabogota.gov.co/sisjur/normas/ Norma1.jsp?i=6739

Comisión Europea (2011). Estrategia renovada de la UE para 2011-2014 sobre responsabilidad social de las empresas. Disponible en: http:// eur-lex.europa.eu/LexUriServ/LexUriServ. do?uri=COM:2011:0681:FIN:ES:PDF

Cooke, Terence E. (1989). Voluntary

Disclosure by Swedish Companies. Journal of International Financial Management and Accounting, 1 (2), 171-195.

Correa-García, Jaime Andrés; Álvarez-Osorio, Martha Cecilia \& Pulgarín-Arias, Andrés Felipe (2011). Evaluación de la calidad en la revelación de la información contable y corporativa para empresas del sector real en los años 2009 y 2010: caso colombiano. 
XVII Congreso Internacional de Contaduría, Administración e Informática. México D.F.: Asociación Nacional de Facultades y Escuelas de Contaduría y Administración. Disponible en: http://congreso.investiga. fca.unam.mx/es/docs/anteriores/xvii/docs/ F02.pdf

Gálvez-Rodríguez, María del Mar; CabaPérez, María del Carmen \& López-Godoy, Manuel (2012). Responsabilidad social y transparencia on-line de las ONG: análisis del caso español. Revista de Economía Pública, Social y Cooperativa, 74, 207-238. Disponible en: http:// www.ciriec-revistaeconomia.es/banco/ CIRIEC_7408_Galvez_et_al.pdf García-Sánchez, Isabel María; RodríguezDomínguez, Luis \& Gallego-Álvarez, Isabel (2011). Corporate Governance and Strategic Information on the Internet. Accounting, Auditing \& Accountability Journal, 24 (4), 471-501.

Global Reporting Initiative, GRI (2006). Guía para la elaboración de memorias de sostenibilidad V. 3.0. Disponible en Global Reporting Initiative: https://www. globalreporting.org, http://www.cyta.com. ar/biblioteca/bddoc/bdlibros/334_gri.pdf Global Reporting Initiative, GRI (2013). Guía para la elaboración de memorias de sostenibilidad V. 4.0. Disponible en Global Reporting Initiative: https:// www.globalreporting.org, https://www. globalreporting.org/resourcelibrary/ Spanish-G4-Part-One.pdf, https://www. globalreporting.org/resourcelibrary/ Spanish-G4-Part-Two.pdf
Gómez, Fabio \& Católico, Diego (2009).

Revelación y divulgación de la información financiera y no financiera on-line de las 500 empresas más representativas en Colombia. Cuadernos de Contabilidad, 10 (27), 269318. Disponible en: http://www.javeriana. edu.co/fcea/cuadernos_contab/vol10_n_27/ vol10_27_3.pdf

Hamburger, Álvaro (2008). Humanización de la empresa. Hacia una ética aplicada en las organizaciones. Bogotá: Universidad de San Buenaventura.

Haro-de Rosario, Arturo; Alarcón-Senent, Francisco \& Caba-Pérez, María del Carmen (2012). Los determinantes de la divulgación de información sobre responsabilidad social corporativa en el caso español. Revista Facultad de Ciencias Económicas: Investigación y Reflexión, 20 (1), 189-206. Disponible en: http://www.scielo.org.co/ pdf/rfce/v20n1/v20n1a12.pdf

International Finance Corporation, IFC (2005).

Estudio de casos de buenas prácticas de gobierno corporativo. Washington, D.C.: International Finance Corporation, IFC. Disponible en: http://www.oecd.org/corporate/ca/ corporategovernanceprinciples/35659647. pdf

International Organization for Standardization, ISO (2004). Norma internacional ISO 14001: sistema de gestión medioambiental. Ginebra: International Organization for Standardization, ISO. Disponible en: https:/www.iso.org/obp/ ui/\#iso:std:iso:14001:ed-2:v1:es

International Organization for Standardization, ISO (2010). Norma Internacional ISO 
REVELACIÓN DE LA RSE EN CÓDIGOS DE BUEN GOBIERNO / L. ROMERO, L. WANUMEN, D. CATÓLICO / 623

26000: guía sobre responsabilidad social.

Ginebra: International Organization

for Standardization, ISO. Disponible

en: https://www.iso.org/obp/

ui/\#iso:std:iso:26000:ed-1:v1:es

Marín-Gaviria, María del Pilar; Maldonado-

García, Stella \& Sanmiguel-Rodríguez, Laura (2012). Análisis empírico de la divulgación de información social en Chile: un estudio en empresas cotizadas. Revista Economía, Gestión y Desarrollo, 13, 121-153. Disponible en: http://revistaeconomia. puj.edu.co/html/articulos/Numero_13/ MALDONADO.pdf

Moneva-Abadía, José Mariano; Ortas-

Fredes, Eduardo \& Acero-Fraile, Isabel (2013). Divulgación de la información de responsabilidad social en las cooperativas de crédito y cajas de ahorros aragonesas. Revista de Economía Pública, Social y Cooperativa, 77, 5-29. Disponible en: http:// www.redalyc.org/pdf/174/17429864001.pdf Morales-Parada, Fernando \& Jarne-Jarne, José Ignacio (2009). E-gobierno corporativo: evidencia en Chile sobre la divulgación de información en internet. Capic Review, 7, 45-62. Disponible en: http://www.capic.cl/ capic/images/vol7tema4.pdf

Organización para la Cooperación y el

Desarrollo Económicos, OCDE (2004).

Principios de gobierno corporativo de la OCDE. París: Organización para la Cooperación y el Desarrollo Económicos, OCDE.

Disponible en: http://www.oecd.org/daf/ca/ corporategovernanceprinciples/37191543. pdf
Pelayo-Velázquez, Marcela de Guadalupe; Fuentes-Callén, Yolanda; CuéllarFernández, Beatriz \& Arias-Uribe, Myriam (2013). Impacto de la divulgación de información financiera en internet de las empresas en América Latina. Global Conference on Business and Finance Proceedings, 8 (1), 772-781. Disponible en: http://www.theibfr.com/ARCHIVE/ISSN1941-9589-V8-N1-2013.pdf

Rodríguez-Domínguez, Luis; Gallego-Álvarez, Isabel \& García-Sánchez, Isabel María (2010). Determinantes de la divulgación voluntaria de información estratégica en internet: un estudio de las empresas españolas cotizadas. Revista Europea de Dirección y Economía de la Empresa, 19 (1), 9-26. Disponible en: http://www.aedemvirtual.com/articulos/126821232100.pdf

Rodríguez-Bolívar, Manuel Pedro \& JusticiaJiménez, Ana María Carolina (2006). Divulgación en internet de los aspectos financieros del gobierno corporativo de las entidades financieras colombianas. Revista Activos, 12, 45-74. Disponible en: http:// contaduria.usta.edu.co/images/documentos/ activos/12-3.pdf

Superintendencia Financiera de Colombia (2007). Circular Externa 028. Código de mejores prácticas corporativas de Colombia. Bogotá D.C.: Superintendencia Financiera de Colombia. Disponible en: https://www. superfinanciera.gov.co/SFCant/Codigopais/ textos/codigopias.pdf

Superintendencia Financiera de Colombia (2011). Circular Externa 007. Código de mejores prácticas corporativas de Colombia. 
Modificación de la Circular Externa 028 de 2007. Bogotá D.C.: Superintendencia Financiera de Colombia. Disponible en: http://www.bolsamercantil.com.co/ Library/News/Files/C\%C3\%B3digo\%20 Pa\%C3\%ADs3417.PDF

United Nations Global Compact (2012). Los diez principios del Pacto Mundial. Disponible en: http://www.unglobalcompact.org/ Languages/spanish/Los_Diez_Principios. html

Vásquez-Paniagua, José Alfredo \& Gonzales-Isaza, Diana Patricia (2009). Metodología para implementar un modelo de responsabilidad social empresarial (RSE) en la industria de la curtiembre en Colombia. Contabilidad y Negocios, 4 (8), 49-56. http://revistas. concytec.gob.pe/pdf/cyn/v4n8/a07v4n8.pdf

- Fecha de recepción: 30 de octubre de 2013

- Fecha de aceptación: 29 de marzo de 2014

- Disponible en línea: 01 de julio de 2014

\section{Para citar este artículo}

Romero-González, Laura Tatiana; WanumenMarín, Luisa Fernanda \& Católico-Segura, Diego Fernando (2014). Revelación de lineamientos sobre responsabilidad social en los códigos de buen gobierno de las empresas cotizadas en Colombia. Cuadernos de Contabilidad, 15 (38), 599-624.

doi: 10.11144/Javeriana.cc15-38.rlsr 